\title{
Proliferaciones mucosas ocasionadas por Gomphoneis minuta (Stone) Kociolek \& Stoermer en ríos de Arequipa, Perú
}

\author{
Mucous proliferations caused by Gomphoneis minuta (Stone) \\ Kociolek \& Stoermer in rivers of the Arequipa, Peru \\ Pablo Franco León ${ }^{1 *}$, Paola Franco Vásquez², \\ Liduvina Sulca Quispe ${ }^{3}$, Viviana Almanza Marroquín ${ }^{4}$
}

\section{RESUMEN}

Por primera vez se encontró la presencia de proliferaciones algales mucosas de color amarillo pardo en los ríos Orcopampa, Caquemayo y Pillones en la provincia de Arequipa, Perú. El agente causante corresponde a Gomphoneis minuta de las floraciones, confirmada por microscopía electrónica de barrido: diatomea con estrías biseriadas, rafe a lo largo de la cara valvar y pedúnculos mucilaginosos en su extremo basal. Se considera como el primer registro para esta zona. Es necesaria la implementación de programas de monitoreo para controlar y evitar su propagación. Además, el hecho de que G. minuta haya sido encontrada en forma considerable en los ríos Orcopampa, Caquemayo y Pillones nos hace presumir que sería capaz de transformarse en un taxón potencialmente nocivo para el ecosistema acuático y que, en forma similar a D. geminata, necesitaría monitoreo y programas de vigilancia para evitar su propagación.

Palabras clave: Gomphoneis minuta, proliferaciones mucosas, Perú.

\section{ABSTRACT}

For the first time, the proliferation of yellow mucus algae was found in the Orcopampa, Caquemayo and Pillones rivers in the province of Arequipa, Peru. The causative agent corresponds to Gomphoneis minuta of the blooms, confirming it by scanning electron microscopy; diatomea with biseriate striae, raphe along the valvar face and muscilaginous peduncles at its basal end, being considered the first record for this area. It is necessary to implement monitoring programs to control and prevent their spread. In addition, the fact that $G$. minuta has been found in considerable extent in the Orcopampa, Caquemayo and Pillones rivers makes us presume that it would be capable of transforming into a taxon potentially harmful to the aquatic ecosystem and, similarly to D. geminata, it would need monitoring and surveillance programs to prevent its spread.

Keywords: Gomphoneis minuta, mucous proliferations, Peru.

\section{Introducción}

Gomphoneis minuta (Stone) Kociolek \& Stoermer, G. eriense (Grunow) Skvortsov \& K.I. Meyer, G. herculeana (Ehrenberg) Cleve, Didymosphenia geminata (Lyngbye) M. Schmidt Cymbella janischii (A. Schmidt) De Toni y Cymbella mexicana (Ehrenberg) Cleve son especies que forman crecimientos conspicuos en las corrientes (Bahls, 2007). Las proliferaciones de pedúnculos pueden cubrir casi todos los sustratos bentónicos disponibles, formando masas mucilaginosas, mucosas y densas de varios centímetros de grosor, y alcanzar hasta $20 \mathrm{~km}$ de extensión (Elwell, 2006). Didymosphenia geminata se ha convertido en un motivo de preocupación mundial, por ser un agresivo invasor de ríos y arroyos en los hemisferios norte y sur debido a su capacidad para formar molestas floraciones en sistemas oligotróficos (Reid y Torres, 2014). Comúnmente conocida como "didymo",

\footnotetext{
Instituto General de Investigación, Universidad Nacional Jorge Basadre Grohmann, Tacna, Perú.

Herbario Takana, Universidad Nacional Jorge Basadre Grohmann, Tacna, Perú.

Laboratorio de Botánica, Facultad de Ciencias, Universidad Nacional Jorge Basadre Grohmann, Tacna, Perú.

Centro de Recursos Hídricos para la Agricultura y Minería (CRHIAM). Universidad de Concepción, Chile.

* Autor por correspondencia: pfrancol@unjbg.edu.pe
}

Fecha de Recepción: 5 de Marzo, 2019.

Fecha de Aceptación: 18 Agosto, 2019. 
"moco de roca" y "alga chapapote" (Díaz et al., 2011), D. geminata es la primera diatomea bentónica no tóxica que causa fuertes efectos negativos en los ambientes acuáticos, aunque muchos aspectos de su biología aún son poco conocidos (Blanco y Ector, 2009). Ríos en Argentina, Chile y Perú son especialmente vulnerables a la invasión de D. geminata (Kumar 2008) y es probable que esto suceda si los protocolos de monitoreo no se implementan.

Gomphoneis minuta muestra capacidad adaptativa a diversidad de hábitats, cuya predilección son cuerpos de agua con alto contenido de materia orgánica, conductividad elevada, $\mathrm{pH}$ alcalino y oxígeno disuelto alto (Kociolek y Stoermer, 1988). Su morfología se caracteriza por valvas robustas, claviformes, heteropolaridad, cabeza que se estrecha gradualmente; estigma único claramente visible en microscopía óptica, rafe lateral y corrugado, con extremos proximales curvados ligeramente en el mismo lado, presencia de líneas longitudinales ubicadas a cada lado y estrías biseriadas (Bey y Ector, 2013). En una primera observación la diatomea se podría confundir con el género Gomphonema, pero se descarta por la presencia de las líneas longitudinales características del género Gomphoneis. Según Kociolek y Stoermer (1988), G. minuta posee valvas de 20-128 $\mu \mathrm{m}$ de largo, 14-26 $\mu \mathrm{m}$ de ancho y 10-12 estrías en $10 \mu \mathrm{m}$.

Gomphoneis minuta fue señalada en Argentina por primera vez entre los años 1989 y 1990 en el curso inferior del río Chubut en Patagonia (Ayestaran y Sastre, 1995). En Chile ha sido señalada desde el año 1971 para el estero Lenga (Rivera et al., 1973), en los ríos Andalién y Biobío, desembocadura (Rivera y Arcos, 1975), en los ríos Laraquete y Carampangue (Rivera y Valdebenito, 1979) y en la Bahía de Coliumo (Rivera y González, 1984). Gomphoneis minuta se ha extendido también en España, donde ha aparecido en algunos ríos del norte en la cuenca del río Ebro (Ortiz-Lerín et al., 2007) y en la cuenca del río Duero (Blanco et al., 2008, Kermarrec et al., 2011). En Francia fue encontrada por primera vez en 1990 en el río Ardèche, afluente del río Ródano (Coste et al., 1992), y en el 2013 en el río Doller, afluente del río Ill y del río Rin, en la región Alsacia (Heudre et al., 2014). Se convirtió en poco tiempo en una molestia para los ríos de ese país, donde se le considera como una especie no indígena (Bey \& Ector, 2013).

Kociolek \& Stoermer (1988) y Johansen et al. (2004) encontraron Gomphoneis minuta predominante en el curso inferior de los ríos y en lagos interiores, desde la Columbia Británica hasta Arizona y del este hasta la costa atlántica. En el río Chikugo, Kyushu, Japón, Gomphoneis minuta fue acompañante de grandes colonias de Cymbella janischii (Suzawa et al., 2011), especies que no han sido registradas en Japón y que probablemente fueron introducidas desde América del Norte. En Chile el estudio de floraciones por D. geminata, realizado por Rivera et al. (2013) en el río Biobío, comprobó que G. minuta fue un componente de la flora acompañante del evento mencionado (Basualto et al., 2016). Se hallaron concentraciones abundantes de G. minuta en los ríos Epuyen y Tecka, también acompañando a proliferaciones $D$. geminata en los ríos Chubut y Azul (Sastre et al., 2014). Reid y Torres (2014) encontraron colonias localizadas de G. minuta junto a las floraciones de Didymosphenia geminata en los ríos Espolón, Coyhaique y Baker de la región de Aysén. Basualto et al. (2016) informan sobre el primer reporte de Didymosphenia geminata y Gomphoneis minuta en el río Laja formando conglomerados masivos de color marrón claro sobre piedras expuestas al torrente de agua.

En el año 2017 se detectaron proliferaciones mucosas color pardo amarillento adheridas a piedras que cubrían extensas áreas en los ríos Orcopampa, Caquemayo y Pillones en la región Arequipa, Perú. Ante tal problemática se desarrolló la presente investigación con el objetivo principal de identificar la diatomea bentónica responsable del evento.

\section{Materiales y métodos}

Las colectas fueron realizadas en marzo del 2017 en el río Orcopampa del distrito de Chilcaymarca a $3.845 \mathrm{msnm}$; y en diciembre del 2017 en los ríos Caquemayo, distrito de Yanque a $4.316 \mathrm{msnm}$, y Pillones, distrito San Antonio de Putina a 4.347 msnm, en Arequipa, Perú (Figura 1). Se establecieron tres estaciones de muestreo en cada uno de los ríos mencionados.

El perifiton fue colectado siguiendo las recomendaciones de Stevenson y Rollins (2007), mediante el cepillado de un área equivalente a $25 \mathrm{~cm}^{2}$ con 5 repeticiones. Las muestras de perifiton fueron fijadas con formol al 4-5\% y etiquetadas señalando el código de la estación, cuerpo de agua de procedencia, nombre de analista y fecha de colecta. Se utilizó la metodología de Battarbee (1986), empleando peróxido de hidrógeno y ácido clorhídrico en el lavado de 


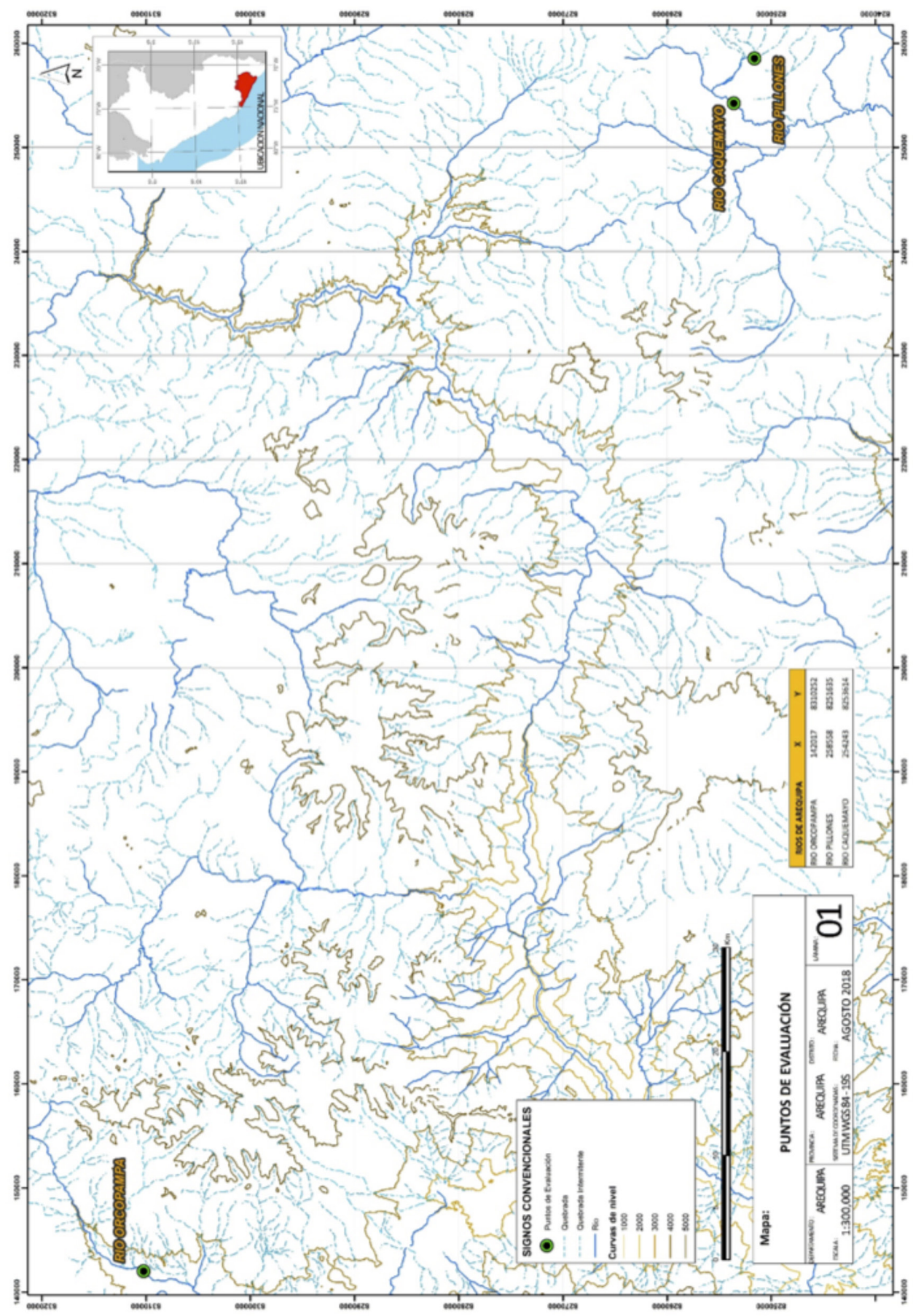

Figura 1. Mapa de ubicación de los ríos Orcopampa, Caquemayo y Pillones, Arequipa, Perú.

frústulos. Se usó la resina NAPHRAX que posee un índice de refracción hasta de 1,73 (Ruhland et al., 1999) para el preparado de montajes permanentes. Los parámetros fisicoquímicos fueron registrados con el equipo multiparámetro portátil marca $\mathrm{HACH}$. Para el cálculo dela calidad del agua (Tabla 1) se utilizó el índice de eutrofización/polución (EPI-D) (Dell'Uomo, 2004): 
Tabla 1. Calidad del agua según

el índice EPI-D (Dell'Uomo, 2004)

\begin{tabular}{ccll}
\hline \multicolumn{1}{c}{ Valor EPI-D } & Clase & Calidad & \multicolumn{1}{c}{ Colores } \\
\hline $0,0<$ EPI-D $<1,0$ & I & Óptimo & Azul \\
$1,0<$ EPI-D $<1,7$ & II & Bueno & Verde \\
$1,7<$ EPI-D $<2,3$ & III & Mediocre & Amarillo \\
$2,3<$ EPI-D $<3,0$ & IV & Mala & Anaranjado \\
$3,0<$ EPI-D $<4,0$ & V & Pésima & Rojo \\
\hline
\end{tabular}

$E P I-D=j=1$ naj.rj.ijj=1naj.rj.

$a_{j}=$ abundancia de la especie $j(\%)$.

$r_{j}=$ fiabilidad de la especie $j$ (5 para indicador óptimo, 3 para buen indicador y 1 para indicador aceptable).

$i_{j}=$ índice de sensibilidad de la especie $j$ (valores de

0 a 4 , que van desde ambiente de óptima calidad a pésima).

Este índice se basa en la sensibilidad (tolerancia/ afinidad) de diatomeas a nutrientes, sustancias orgánicas y grado de mineralización, clasificando los cuerpos de agua en 5 clases de calidad.

La observación de las muestras biológicas se realizó mediante el uso de microscopía óptica Micros Austria y electrónica de barrido de marcas Tescan
Vega II. Las dimensiones fueron medidas en el microscopio de barrido y la abundancia de especies se calculó mediante la cámara de Sedgewick Rafter cuadriculada, empleando claves de identificación de Bey y Ector (2013).

\section{Resultados}

En una extensión de 500 metros en el río Orcopampa (Figura 2a) y de 250 metros para los ríos Caquemayo y Pillones, se halló que la mayoría de piedras expuestas estaban cubiertas de masas mucosas color amarillo pardo con un espesor máximo de $2,5 \mathrm{~cm}$ (Figura 2a-g). Las muestras biológicas fueron ingresadas bajo la codificación R-ORC01, R-ORC02, R-ORC03 (río Orcopampa); R-CAQ01, R-CAQ02, R-CAQ03 (río Caquemayo) y R-PILL01, R-PILLO02, R-PILL03 (río Pillones), a la colección del Herbario Takana, Facultad de Ciencias, Universidad Nacional Jorge Basadre Grohmann, Tacna, Perú.

La calidad para las estaciones (Tabla 2) del río Orcopampa fue mala, pésima y pésima; para el Caquemayo fue mala, mediocre y mediocre; y Pillones fue mala, pésima y mediocre. Los parámetros fisicoquímicos (Tabla 3) señalan un pH máximo de 8,90 indicando aguas alcalinas.

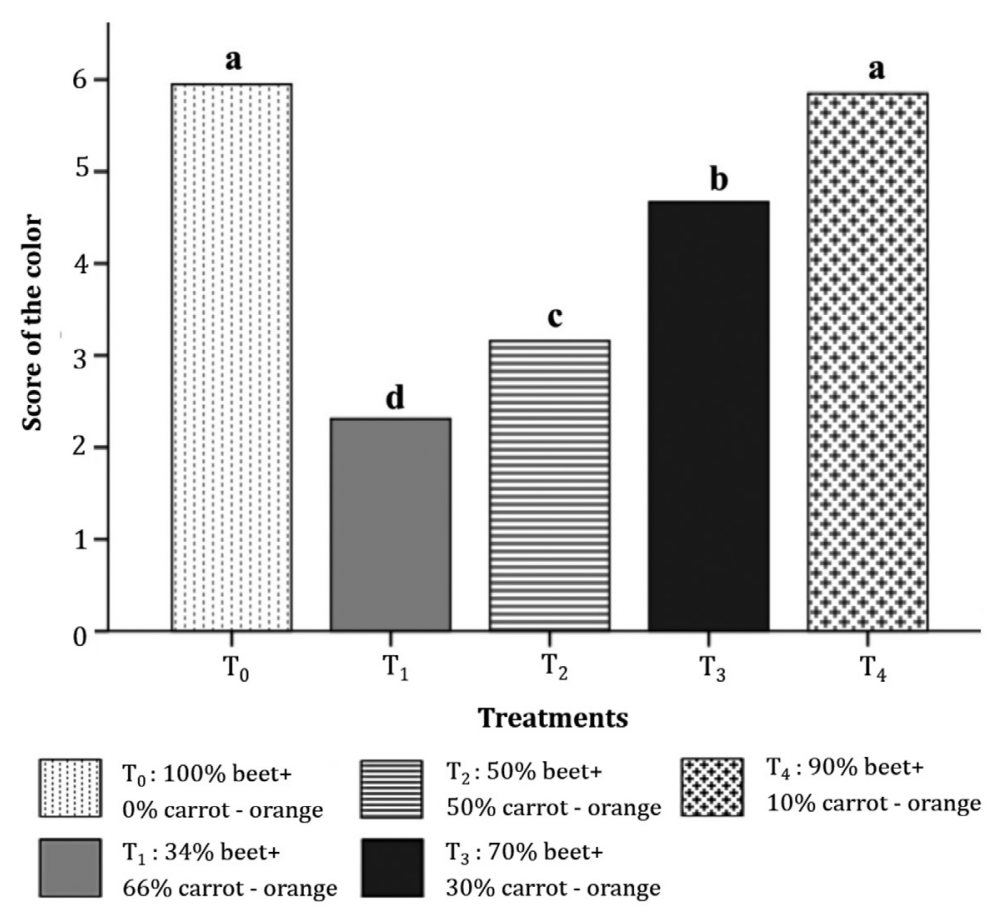

Figura 2. a. Vista general del río Orcopampa. b, c y d. Aspecto de los conglomerados masivos por Gomphoneis minuta sobre piedras en el río Orcopampa. 
Tabla 2. Cálculo de la calidad de agua mediante el índice EPI-D según Dell'Uomo (2004) (O = óptimo, B: bueno, $\mathrm{Me}=$ mediocre, $\mathrm{M}=$ mala y $\mathrm{P}=$ pésima) de los ríos Orcopampa, Caquemayo y Pillones.

\begin{tabular}{lllll}
\hline \multirow{2}{*}{ Puntos de muestreo } & \multicolumn{3}{c}{ EPI-D } \\
\cline { 2 - 5 } & & Valor & Calidad & \multicolumn{1}{c}{ Color } \\
\hline \multirow{2}{*}{ Río Orcopampa } & R-ORC01 & 2,7 & Mala & Anaranjado \\
\cline { 2 - 5 } & R-ORC02 & 3,3 & Pésima & Rojo \\
\cline { 2 - 5 } R-ORC03 & 3,3 & Pésima & Rojo \\
\hline \multirow{2}{*}{ Río Caquemayo } & R-CAQ01 & 2,3 & Mala & Anaranjado \\
\cline { 2 - 5 } & R-CAQ02 & 2,0 & Mediocre & Amarillo \\
\cline { 2 - 5 } Río Pillones & R-CAQ03 & 1,9 & Mediocre & Amarillo \\
\cline { 2 - 5 } & R-PILL01 & 2,1 & Mediocre & Amarillo \\
\cline { 2 - 5 } & R-PILL02 & 3,6 & Pésima & Rojo \\
\hline
\end{tabular}

Los géneros en la comunidad fitoplanctónica (Tabla 4) fueron Achnanthes, Achnanthidium, Asterionella, Aulacoseira, Cocconeis, Craticula, Cyclotella, Cymbella, Denticula, Diadesmis, Encyonema, Epithemia, Fragilaria, Frustulia, Gomphoneis, Hannaea, Luticola, Melosira, Navicula, Neidiium, Nitzschia, Pinnularia, Reimeria, Rhoicosphenia, Sellaphora, Surirella, Synedra y Ulnaria.

El estudio de las muestras biológicas evidenció que la especie causante de las proliferaciones mucosas fue Gomphoneis minuta formando abundantes pedúnculos mucilaginosos (Figura 4a), por la presencia de estrías biseriadas, estigma único (Figura 4c). La dimensión promedio (Tabla 5) de G. minuta fue $40,82 \mu \mathrm{m}$ y $11,9 \mu \mathrm{m}$.
La abundancia de Gomphoneis minuta en el perifiton promedio fue de $421 \mathrm{cel} / \mathrm{mm}^{2}, 7.144 \mathrm{cel} /$ $\mathrm{mm}^{2}$ y $40.394 \mathrm{cel} / \mathrm{mm}^{2}$ (río Orcopampa) $250 \mathrm{cel} / \mathrm{ml}$, $200 \mathrm{cel} / \mathrm{ml}$ y $53 \mathrm{cel} / \mathrm{ml}$ (río Caquemayo) y $180 \mathrm{cel} /$ $\mathrm{ml}, 224 \mathrm{cel} / \mathrm{ml}$ y $72 \mathrm{cel} / \mathrm{ml}$ (río Pillones).

\section{Discusión}

Es la primera vez que se reporta en Perú este tipo de floramiento algal debido a Gomphoneis minuta en cuerpos de agua. La importancia del río Orcopampa radica en la crianza de truchas para las poblaciones humanas aledañas y la de los ríos Pillones y Caquemayo en su uso para los embalses de agua potable. G. minuta aparentemente aumenta su proliferación en $\mathrm{pH}$ alcalino. En Chile se reportaron concentraciones

Tabla 3. Parámetros fisicoquímicos de las estaciones de muestreo.

\begin{tabular}{lccccr}
\hline \multirow{2}{*}{ Ríos } & \multirow{2}{*}{ Estaciones de muestreo } & \multicolumn{4}{c}{ Parámetros fisicoquímicos } \\
\cline { 2 - 6 } & & $\mathrm{pH}$ & Oxígeno disuelto & Temperatura & Conductividad \\
\hline \multirow{3}{*}{ Río Orcopampa } & R-ORC01 & 8,90 & $7,52 \mathrm{mg} / \mathrm{L}$ & $6,1{ }^{\circ} \mathrm{C}$ & $105,7 \mu \mathrm{S} / \mathrm{cm}$ \\
\cline { 2 - 6 } & R.ORC02 & 8,89 & $7,47 \mathrm{mg} / \mathrm{L}$ & $6,0{ }^{\circ} \mathrm{C}$ & $103,6 \mu \mathrm{S} / \mathrm{cm}$ \\
\cline { 2 - 6 } & R-ORC03 & 8,86 & $7,69 \mathrm{mg} / \mathrm{L}$ & $6,1{ }^{\circ} \mathrm{C}$ & $98,2 \mu \mathrm{S} / \mathrm{cm}$ \\
\hline \multirow{2}{*}{ Río Caquemayo } & R-CAQ01 & 8,85 & $7,48 \mathrm{mg} / \mathrm{L}$ & $6,3^{\circ} \mathrm{C}$ & $98,9 \mu \mathrm{S} / \mathrm{cm}$ \\
\cline { 2 - 6 } & R-CAQ02 & 8,81 & $7,34 \mathrm{mg} / \mathrm{L}$ & $6,8^{\circ} \mathrm{C}$ & $102,5 \mu \mathrm{S} / \mathrm{cm}$ \\
\hline \multirow{nyyyyy}{*}{ Río Pillones } & R-CAQ03 & 7,76 & $7,86 \mathrm{mg} / \mathrm{L}$ & $7,0^{\circ} \mathrm{C}$ & $93,4 \mu \mathrm{S} / \mathrm{cm}$ \\
\cline { 2 - 6 } & R-PILL01 & 8,87 & $7,35 \mathrm{mg} / \mathrm{L}$ & $6,4^{\circ} \mathrm{C}$ & $100,5 \mu \mathrm{S} / \mathrm{cm}$ \\
\cline { 2 - 6 } & R-PILL02 & 8,01 & $7,86 \mathrm{mg} / \mathrm{L}$ & $6,7{ }^{\circ} \mathrm{C}$ & $96,7 \mu \mathrm{S} / \mathrm{cm}$ \\
\hline
\end{tabular}


Tabla 4. Comunidad fitoplanctónica de los ríos Orcopampa, Caquemayo y Pillones.

\begin{tabular}{|c|c|c|c|c|c|c|c|c|c|}
\hline \multirow{2}{*}{ Diatomeas } & \multicolumn{3}{|c|}{ Río Orcopampa } & \multicolumn{3}{|c|}{ Río Caquemayo } & \multicolumn{3}{|c|}{ Río Pillones } \\
\hline & ORC01 & ORC02 & ORC03 & CAQ01 & CAQ02 & CAQ03 & PILL01 & PILL02 & PILL03 \\
\hline Achnanthes lanceolata & & & & & $\mathrm{x}$ & & & & \\
\hline Achnanthidium sp. & $\mathrm{x}$ & $\mathrm{x}$ & $X$ & & & & & & \\
\hline Asterionella sp. & & $\mathrm{x}$ & $X$ & & & & & & \\
\hline Aulacoseira sp. & & $\mathrm{x}$ & $\mathrm{X}$ & & & $\mathrm{x}$ & & & \\
\hline Cocconeis sp. & $\mathrm{x}$ & $\mathrm{x}$ & $\mathrm{X}$ & & & & & & $\mathrm{x}$ \\
\hline Craticula halophila & & & & & & & & $\mathrm{x}$ & \\
\hline Cyclotella sp. & & $\mathrm{x}$ & $X$ & & & & & & \\
\hline Cymbella sp. & & $\mathrm{x}$ & $\mathrm{X}$ & & $\mathrm{x}$ & & & & \\
\hline Denticula sp. & & & & & $\mathrm{x}$ & & $\mathrm{x}$ & & \\
\hline Diadesmis sp. & & & & & & & $\mathrm{x}$ & & \\
\hline Encyonema sp. & $\mathrm{x}$ & & & & $\mathrm{x}$ & $\mathrm{x}$ & $\mathrm{x}$ & $\mathrm{x}$ & $\mathrm{x}$ \\
\hline Epithemia sp. & & $\mathrm{x}$ & & & & & & & \\
\hline Fragilaria crotonensis & & & $\mathrm{X}$ & & & $\mathrm{x}$ & & & \\
\hline Fragilaria sp. & & $\mathrm{x}$ & & & & & & & \\
\hline Frustulia franguelii & & & & $\mathrm{x}$ & & $\mathrm{x}$ & $\mathrm{x}$ & & $\mathrm{x}$ \\
\hline Frustulia saxonica & & & & $\mathrm{x}$ & $\mathrm{x}$ & $\mathrm{x}$ & $\mathrm{x}$ & $\mathrm{x}$ & \\
\hline Gomphoneis minuta & $\mathrm{x}$ & $\mathrm{x}$ & $X$ & & & & & & \\
\hline Hannaea sp. & $\mathrm{x}$ & $\mathrm{x}$ & $X$ & & & & & & \\
\hline Luticola sp. & $\mathrm{x}$ & & & & & $\mathrm{x}$ & & $\mathrm{x}$ & $\mathrm{x}$ \\
\hline Melosira sp. & & & & & & & $\mathrm{x}$ & & \\
\hline Navicula sp. & $\mathrm{x}$ & $\mathrm{x}$ & & $\mathrm{x}$ & & & $\mathrm{x}$ & & \\
\hline Neidiium sp. & & & & $\mathrm{x}$ & & & & $\mathrm{x}$ & $\mathrm{x}$ \\
\hline Nitzschia sp. & $\mathrm{x}$ & $\mathrm{x}$ & $X$ & & & & & & \\
\hline Pinnularia sp. & & & & $\mathrm{x}$ & & $\mathrm{x}$ & $\mathrm{x}$ & $\mathrm{x}$ & \\
\hline Reimeria sp. & $\mathrm{x}$ & $\mathrm{x}$ & $X$ & & & & & & \\
\hline Rhoicosphenia sp. & $\mathrm{x}$ & $\mathrm{x}$ & $\mathrm{X}$ & & & & & & \\
\hline Sellaphora sp. & & & & $\mathrm{x}$ & & & & $\mathrm{X}$ & \\
\hline Surirella sp. & & & & & $\mathrm{x}$ & $\mathrm{x}$ & $\mathrm{x}$ & & \\
\hline Synedra radians & & & & & & & $\mathrm{x}$ & & \\
\hline Ulnaria $s p$ & $\mathrm{x}$ & $\mathrm{x}$ & $X$ & & $\mathrm{x}$ & & & & \\
\hline
\end{tabular}

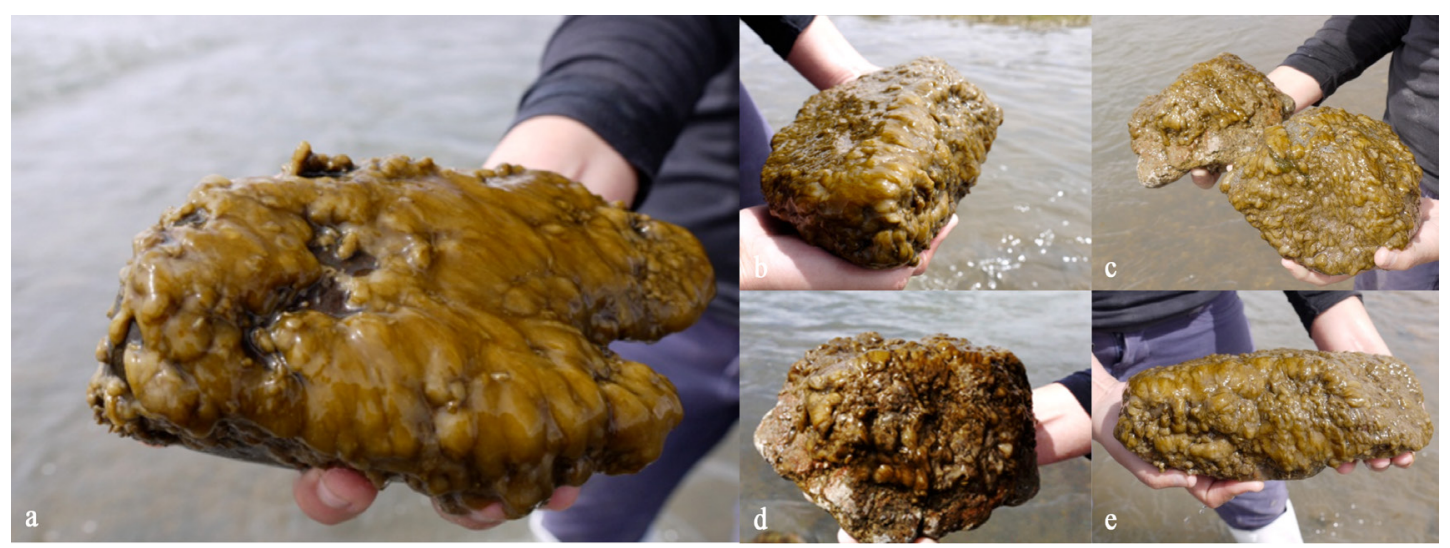

Figura 3. Aspecto de los conglomerados masivos por Gomphoneis minuta en los ríos Caquemayo (a, b y c) y Pillones (e).

abundantes de G. minuta en los ríos Epuyen y Tecka, y acompañando a proliferaciones considerables de D. geminata en los ríos Chubut y Azul (Sastre et al.,
2014). Floraciones masivas de $D$. geminata y G. minuta se encontraron en el río Laja (Basualto et al., 2016). Las proliferaciones por $D$. geminata son similares a 


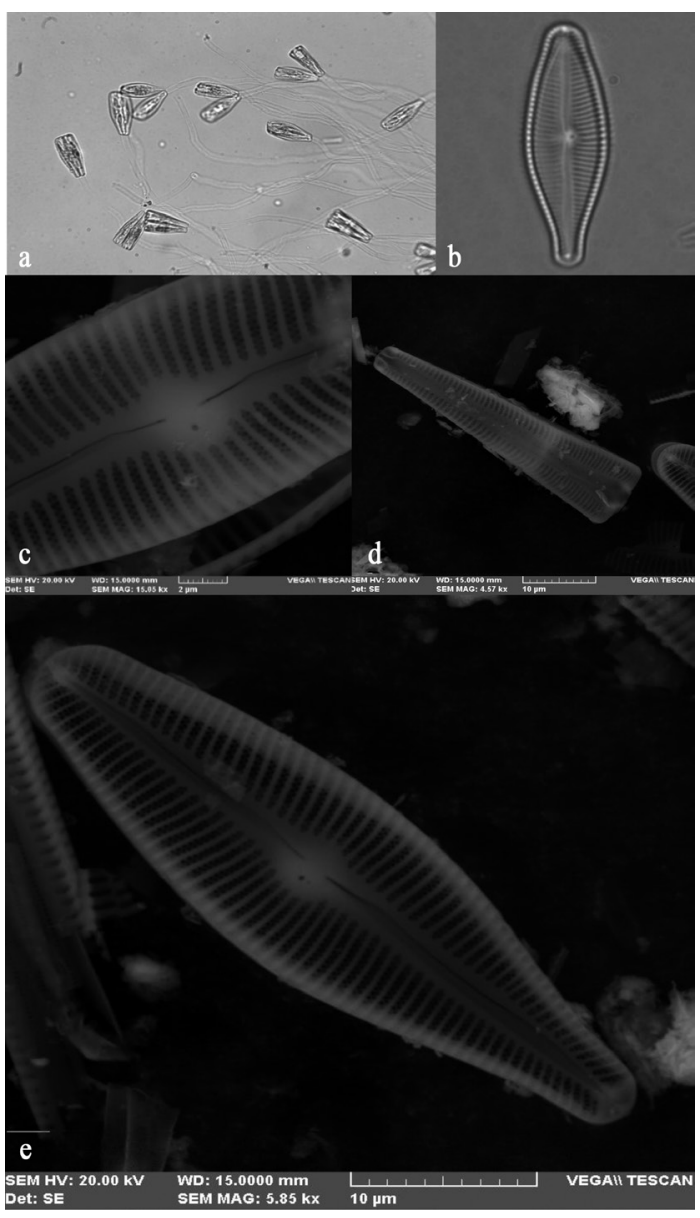

Figura 4. a. Pedúnculos mucilaginosos de Gomphoneis minuta (40x). b. Gomphoneis minuta (100x). c. Estrías biseri adas y estigma (15000x). d. Vista lateral (4000x). e. Gomphoneis minuta (5000x).

las causadas por G. minuta, dado su aspecto mucoso, llegando a confundirse.

Además, el hecho de que G. minuta haya sido encontrada en forma considerable en los ríos Orcopampa, Caquemayo y Pillones nos hace
Tabla 5. Dimensiones de individuos de Gomphoneis minuta.

\begin{tabular}{|c|c|c|}
\hline \multirow{2}{*}{ Individuo } & \multicolumn{2}{|c|}{ Dimensiones } \\
\hline & Largo $(\mu \mathrm{m})$ & Ancho $(\mu \mathrm{m})$ \\
\hline 1 & 47,64 & 11,26 \\
\hline 2 & 43,42 & 12,02 \\
\hline 3 & 41,32 & 12,45 \\
\hline 4 & 37,41 & 11,08 \\
\hline 5 & 42,97 & 12,14 \\
\hline 6 & 37,27 & 11,19 \\
\hline 7 & 40,21 & 11,42 \\
\hline 8 & 36,94 & 11,68 \\
\hline 9 & 45,58 & 12,24 \\
\hline 10 & 40,79 & 12,24 \\
\hline 11 & 38,5 & 12,24 \\
\hline 12 & 45,07 & 13,07 \\
\hline 13 & 43,71 & 13,1 \\
\hline 14 & 43,26 & 11,93 \\
\hline 15 & 40,53 & 11,92 \\
\hline 16 & 43,24 & 11,01 \\
\hline 17 & 41,91 & 12,31 \\
\hline 18 & 41,26 & 11,49 \\
\hline 19 & 43,3 & 11,58 \\
\hline 20 & 39,51 & 11,5 \\
\hline 21 & 41,45 & 12,83 \\
\hline 22 & 35,2 & 11,38 \\
\hline 23 & 33,95 & 11,97 \\
\hline 24 & 42,49 & 11,5 \\
\hline 25 & 37,41 & 11,58 \\
\hline 26 & 37,8 & 12,52 \\
\hline 27 & 42,76 & 12,05 \\
\hline 28 & 45,42 & 12,11 \\
\hline 29 & 40,25 & 11,96 \\
\hline 30 & 36,18 & 11,29 \\
\hline 31 & 39,3 & 12,4 \\
\hline 32 & 40,35 & 11,55 \\
\hline
\end{tabular}

presumir que sería capaz de transformarse en un taxón potencialmente nocivo para el ecosistema acuático y que, en forma similar a $D$. geminata, necesitaría monitoreo y programas de vigilancia para evitar su propagación.

\section{Literatura Citada}

Ayestaran, M.G.; Sastre, A.V.

1995. Diatomeas del curso inferior del río Chubut (Patagonia Argentina). Pennales I: Naviculaceae. Boletín de la Sociedad Argentina de Botánica, 31(1-2): 57-68.

Bahls, L.L.

2007. Cymbella janischii - Giant endemic diatom of the Pacific Northwest: morphology, ecology and distribution compared to Cymbella mexicana. Northwest Science, 81: 284-292.
Basualto, S.; Rivera, P.; Cruces, F.; Ector, L.; Ascencio, E. 2016. Didymosphenia geminata (Lyngbye) M. Schmidt y Gomphoneis minuta (Stone) Kociolek \& Stoermer (Bacillariophyta), especies presentes en ríos de la VIII Región, Chile, formadoras de proliferaciones con aspecto muy similar. Gayana Botánica, 73(2): 457-461.

Battarbee, R.W.

1986. Diatom Analysis; in: B.E Berglund (ed.). Handbook of Holocene palaeoecology and palaeohydrology. John Wiley \& Sons. NJ, US. pp. 527-570. 
Bey, M.; Ector, L.

2013. Atlas des diatomées des cours d'eau de la région Rhône-Alpes. Tome 5. Naviculacées: Cymbelloidées, Gomphonématoidées. Direction Régionale de l'Environnement, de l'Aménagement et du Logement. Rhône-Alpes, Lyon. pp. $751-971$.

Blanco, S.; Ector, L.; Huck, V.; Monnier, O.; Cauchie, H.M.;

Hoffmann, L.; E. Bécares.

2008. Diatom assemblages and water quality assessment in the Duero basin (NW Spain). Belgian Journal of Botany, 141(1): 39-50.

Blanco, S.; Ector, L.

2009. Distribution, ecology and nuisance effects of the freshwater invasive diatom Didymosphenia geminata (Lyngbye) M. Schmidt: a literature review. Nova Hedwigia, 88(3-4): 347-422.

Dell'Uomo, A.

2004. L'indice Diatomico di Eutrofizzazione/Polluzione (EPI-D) nel Monitoraggio delle acque Correnti: Linee Guida. Roma. 107 p.

Díaz, C.; Molina J.; Montecino, V.

2011. Manual para el Monitoreo e Identificación de la Microalga Bentónica Didymosphenia geminata. Subsecretaría de Pesca. Gobierno de Chile. 74 p.

Elwell, L.

2006. Increase in nuisance blooms and geographic expansion of the freshwater diatom Didymosphenia geminata. U.S. Geological Survey Open File Report 2007-1425. 38 p.

Heudre, D.; Moreau L.; Ector L.; Wetzel C.E.

2014. Arrivée et propagation d'espèces néobiotiques sur le bassin Rhin- Meuse: une première approche de 1997 à 2013. En: Serieyssol, K.; Beauger, A., Ector, L. (eds.), 33ème Colloque de l'Association des Diatomistes de Langue Française, 7-10 octobre 2014. 55 p.

Johansen, J.R.; Lowe, R.; Gomez, S.R.; Kociolek, J.P. and Masosky, S.A.

2004. New algal species records for the Great Smoky Mountains National Park, U.S.A., with an annotated checklist of all reported species for the park. Algological Studies 111:17-44.

Kermarrec, L.; Ector; L.; Bouchez; A.; Rimet; F.; Hoffmann L. 2011. A preliminary phylogenetic analysis of the Cymbellales based on $18 \mathrm{~S}$ rDNA gene sequencing. Diatom Research, 26(3): 305-315.

Kociolek, J.P.; Stoermer, E.F.

1988. Taxonomy, ultrastructure and distribution of Gomphoneis herculeana, G. eriense and closely related species (Naviculales: Gomphonemataceae). Proceedings of the Academy of Natural Sciences of Philadelphia, 140(2): 24-97.
Kumar, S.

2008. Modeling potential habitat distribution for freshwater diatom Didymosphenia geminata in the continental United States. 93rd ESA Annual Meeting, Comm. no. COS 27-5. Milwaukee. 48 p.

Ortiz-Lerín, R.; Durán, C.; Pardos, M.; Cambra J.

2007. Red de diatomeas en la cuenca del Ebro: Evaluación de la calidad biológica del agua y estudio florístico de las comunidades durante el periodo 2005 y 2006. Algas, 38: 10 .

Reid, B.; Torres, R.

2014. Didymosphenia geminata invasion in South America: Ecosystem impacts and potential biogeochemical state change in Patagonian rivers. Acta Oecologica 54: 101-109.

Rivera, P.; Parra O.; González M.

1973. Fitoplancton del estero Lenga, Chile. Gayana Botánica, 23: 1-93.

Rivera, P. y Arcos D.

1975. Diatomeas más comunes en la desembocadura del río BioBío. Boletín de la Sociedad de Biología de Concepción, 49: 223-230.

Rivera, P.; Valdebenito H.

1979. Diatomeas recolectadas en las desembocaduras de los ríos Chivilingo, Laraquete y Carampangue, Chile. Gayana Botánica 35: 1-99.

Rivera, P.; González M.

1984. Microalgas de los colectores de larvas de mitílidos en Bahía Coliumo, Chile. Phycologia Latino-Americana, 2: $27-81$.

Rivera, P.; Basualto, S.; Cruces, F.

2013. Acerca de la diatomea Didymosphenia geminata (Lyngbye) M. Schmidt: su morfología y distribución en Chile. Gayana Botánica, 70(1): 154-158.

Ruhland, K.; Karst, T.; Paterson, A.; Gregory-Eaves, R.; Smol, J.P.; Cumming B.F.

1999. Paleoecological Environmental Assessment and Research Laboratory Department of Biology Queen's University Standard Sediment Sample Preparation Methods for Siliceous Microfossils (Diatoms and Chrysophyte Scales and Cysts). 25 p.

Stevenson, R.J.; Rollins, S.L.

2007. Ecological Assessments with Benthic Algae. Methods in Stream Ecology. Elsevier Academic Press. pp. 785-803.

Suzawa, T.; Seino, S.; Mayama, S.

2011. Blooms of Cymbella janischii (A.W. Schmidt) De Toni accompanied by Gomphoneis minuta (Stone) Kociolek \& Stoermer from the upper stream of the Chikugo River, Kyushu, Japan, possibility of new alien diatom species. Diatom, 27: 58-64. 